\title{
Cross-Product Cubature Error Bounds
}

\author{
By Frank G. Lether
}

\begin{abstract}
This paper is concerned with cross-product cubature rules. We use Sard's Kernel Theorem [10] to express the cross-product cubature error in terms of one variable kernels. This simplified representation of the error is then used to derive cubature error bounds analogous to those obtained by Secrest and Stroud [13], for quadrature rules.
\end{abstract}

1. Introduction. The problem of finding error bounds for numerical integration rules in two or more dimensions has, until recently, received little attention. The purpose of this paper is to apply a kernel theorem due to Sard [10] to derive error bounds for cross-product cubature rules. Numerical examples, which illustrate the theory, are included.

The cubature error bounds which occur in the literature are of two types: derivative and derivative-free. The derivative-free error bounds are obtained by complexvariable arguments and assume the integrand is analytic in a suitable region. We mention the work of Ahlin [1], Chawla [3] and Stenger [12] for Gaussian crossproduct rules. Barnhill [2] has generalized some of Davis's [4] one-dimensional work to cubature rules. The derivative cubature error bounds give the error in terms of certain partial derivatives of the function to be integrated. Derivative error representations for cross-product rules have been obtained by several authors [6], [8], and [11].

In [13], Stroud and Secrest obtain quadrature error bounds by using Peano's Theorem [5, p. 70]. Their bounds are written as a product of the $L_{1}$ norm of the Peano kernel and a bound on the derivative of the function to be integrated. It is shown in [13] how error bounds for cross-product rules can be obtained from quadrature error bounds by using a method due to Nikol'skii [9]. Sard [10] has generalized Peano's Theorem to the multivariable case. By using Sard's Kernel Theorem [10, p. 200], error bounds analogous to those in [13] can be derived for cubature rules.

In this paper we consider cross-product cubature error bounds. Our basic tool is Sard's Kernel Theorem. We show for cross-product rules that all of the kernels in Sard's error representation are completely determined by the Peano kernels for the quadrature rules which generate the cross-product rule. The Sard kernels for Gaussian rules are shown to have special sign properties which facilitate the calculation of their norms. The error bounds we derive for cross-product rules will be extended to fully symmetric cubature rules in a future paper.

2. The Cubature Error Representation. Let the two quadrature rules

$$
\int_{a}^{b} u(x) r(x) d x=\sum_{\mu=1}^{m} A_{\mu} r\left(a_{\mu}\right)+R(r)
$$

Received July 30, 1969, revised December 22, 1969.

AMS Subject Classifications. Primary 6555; Secondary 4144, 6580.

Key Words and Phrases. Sard kernels, Gaussian rules, Peano kernels, cross-product rules, cubature error bounds. 
and

$$
\int_{c}^{d} v(y) s(y) d y=\sum_{\nu=1}^{n} B_{v} s\left(b_{\nu}\right)+S(s)
$$

have algebraic precision $p$ and $q$, respectively. Let the nodes and weight functions satisfy the following properties:

(i) $a_{\mu} \in[a, b], 1 \leqq \mu \leqq m$ and $\int_{a}^{b} u(x) d x \neq 0$,

(ii) $b_{v} \in[c, d], 1 \leqq \nu \leqq n$ and $\int_{c}^{d} v(y) d y \neq 0$.

We wish to study the cubature error $R \times S(f)$ in the cross-product rule generated by (2.1) and (2.2):

$$
R \times S(f)=\int_{c}^{d} \int_{a}^{b} u(x) v(y) f(x, y) d x d y-\sum_{\nu=1}^{n} \sum_{\mu=1}^{m} A_{\mu} B_{\nu} f\left(a_{\mu}, b_{\nu}\right) .
$$

Let $\alpha \in[a, b], \beta \in[c, d], I=[a, b] \times[c, d]$ and let $\rho$ and $\sigma$ be integers such that $1 \leqq \rho \leqq p+1,1 \leqq \sigma \leqq q+1$. Denote by $C_{\rho, \sigma}(I)$ the space of all real-valued functions $f$ for which the partial derivatives

$$
f_{i, i}=\frac{\partial^{i+i} f}{\partial x^{i} \partial y^{i}}, \quad 0 \leqq i \leqq \rho, 0 \leqq j \leqq \sigma,
$$

are continuous on $I$. Define the functions $\theta$ and $\psi$ as follows:

$$
\begin{array}{rlrl}
\theta(\tilde{x}, x) & =0, & & \tilde{x}<x, \\
& =1, \quad \tilde{x} \geqq x,
\end{array}
$$

and $\psi(\xi, x, x)=\theta(\tilde{x}, \xi)-\theta(\tilde{x}, x)$, where $\xi$ is a real number. Let

$$
\begin{aligned}
(x-\xi)^{(i)} & =(x-\xi)^{i} / j !, & j & =1,2, \cdots, \\
& =1, & j & =0 .
\end{aligned}
$$

We work in the space $C_{\rho, \sigma}(I)$. Since $C_{\rho, \sigma}(I)=B_{\ulcorner\rho, \sigma]}[10$, p. 184, Table 2, p. 207], it follows from Sard's Kernel Theorem 84 [10, p. 200] that

$$
\begin{aligned}
R \times S(f)= & \sum_{i<\rho ; j<\sigma} C^{i, j} f_{i, j}(\alpha, \beta)+\sum_{i<\sigma} \int_{a}^{b} f_{\rho, j}(x, \beta) K^{\rho, j}(x) d x \\
& +\sum_{i<\rho} \int_{c}^{d} f_{i, \sigma}(\alpha, y) K^{i, \sigma}(y) d y+\int_{c}^{d} \int_{a}^{b} f_{\rho, \sigma}(x, y) K^{\rho, \sigma}(x, y) d x d y,
\end{aligned}
$$

where

$$
\begin{array}{rlrl}
C^{i, i} & =R \times S\left[(x-\alpha)^{(i)}(y-\beta)^{(i)}\right], & & i<\rho, j<\sigma, \\
K^{\rho, i}(\tilde{x}) & =R \times S\left[(x-\tilde{x})^{(\rho-1)} \psi(\alpha, \tilde{x}, x)(y-\beta)^{(i)}\right], & & j<\sigma, \\
K^{i, \sigma}(\tilde{y}) & =R \times S\left[(x-\alpha)^{(i)}(y-\tilde{y})^{(\sigma-1)} \psi(\beta, \tilde{y}, y)\right], & i<\rho, \\
K^{\rho, \sigma}(\tilde{x}, \tilde{y}) & =R \times S\left[(x-\tilde{x})^{(\rho-1)} \psi(\alpha, \tilde{x}, x)(y-\tilde{y})^{(\sigma-1)} \psi(\beta, \tilde{y}, y)\right] .
\end{array}
$$

We now show that it is possible to write all of the kernels $K^{p, i}, K^{i, \sigma}$ and $K^{p, \sigma}$ in terms of the two kernels $K^{p, 0}$ and $K^{0, \sigma}$.

Let $P_{k}$ denote the class of all polynomials of degree less than or equal to $k$. The following two lemmas are a direct consequence of (2.3). 
Lemma 1. If $r \in P_{p}$, then

$$
R \times S[r(x) s(y)]=S[s(y)] \int_{a}^{b} u(x) r(x) d x .
$$

Lemma 2. If $s \in P_{a}$, then

$$
R \times S[r(x) s(y)]=R[r(x)] \int_{c}^{d} v(y) s(y) d y .
$$

THEOREM 1. If $f \in C_{\rho . \sigma}(I)$, then

$$
\begin{aligned}
R \times S(f)= & \sum_{i<\sigma} \int_{a}^{b} f_{\rho, i}(x, \beta) K^{\rho, j}(x) d x+\sum_{i<\rho} \int_{c}^{d} f_{i, \sigma}(\alpha, y) K^{i, \sigma}(y) d y \\
& +\int_{c}^{d} \int_{a}^{b} f_{\rho, \sigma}(x, y) K^{\rho, \sigma}(x, y) d x d y,
\end{aligned}
$$

where

$$
\begin{aligned}
K^{\rho, 0}(\tilde{x}) & =-R\left[(x-\tilde{x})^{(\rho-1)} \theta(\tilde{x}, x)\right] \int_{c}^{d} v(y) d y, \\
K^{\rho, j}(\tilde{x}) & =\frac{K^{\rho, 0}(\tilde{x})}{\int_{c}^{d} v(y) d y} \int_{c}^{d} v(y)(y-\beta)^{(j)} d y, \quad 1 \leqq j<\sigma, \\
K^{0, \sigma}(\tilde{y}) & =\text { dual of } K^{\rho, 0}(\tilde{x}), \\
K^{i, \sigma}(\tilde{y}) & =\text { dual of } K^{\rho, j}(\tilde{x}), \quad 1 \leqq i<\rho, \\
K^{\rho, \sigma}(\tilde{x}, \tilde{y}) & =\frac{K^{0, \sigma}(\tilde{y})}{\int_{a}^{b} u(x) d x} F^{\rho, 0}(\tilde{x}, \alpha) \\
& +\frac{K^{\rho, 0}(\tilde{x})}{\int_{c}^{d} v(y) d y} G^{0, \sigma}(\tilde{y}, \beta)-\frac{K^{\rho, 0}(\tilde{x}) K^{0, \sigma}(\tilde{y})}{\int_{a}^{b} u(x) d x \int_{c}^{d} v(y) d y},
\end{aligned}
$$

and

$$
\begin{aligned}
& F^{\rho, 0}(\tilde{x}, \alpha)=\theta(\tilde{x}, \alpha) \int_{a}^{b} u(x)(x-\tilde{x})^{(\rho-1)} d x-\int_{a}^{\tilde{z}} u(x)(x-\tilde{x})^{(\rho-1)} d x, \\
& G^{0, \sigma}(\tilde{y}, \beta)=\text { dual of } F^{\rho, 0}(\tilde{x}, \alpha) .
\end{aligned}
$$

Proof. Since $i<\rho \leqq p+1$, Lemma 1 and (2.5) give

$$
C^{i, i}=0, \quad i<\rho, j<\sigma .
$$

We now calculate $K^{\rho, i}$. By (2.5) and Lemma 2 ,

$$
K^{\rho, i}(\tilde{x})=R\left[(x-\tilde{x})^{(\rho-1)} \psi(\alpha, \tilde{x}, x)\right] \int_{c}^{d} v(y)(y-\beta)^{(j)} d y .
$$


The definition of $\psi$ implies

$$
R\left[(x-\tilde{x})^{(p-1)} \psi(\alpha, \tilde{x}, x)\right]=-R\left[(x-\tilde{x})^{(p-1)} \theta(\tilde{x}, x)\right] .
$$

If we set $j=0$ in (2.6) we obtain

$$
K^{\rho, 0}(\tilde{x})=R\left[(x-\tilde{x})^{(\rho-1)} \psi(\alpha, \tilde{x}, x)\right] \int_{c}^{d} v(y) d y .
$$

The previous relation and (2.7) imply

$$
K^{p, 0}(\tilde{x})=-R\left[(x-\tilde{x})^{(p-1)} \theta(\tilde{x}, x)\right] \int_{0}^{d} v(y) d y .
$$

Relations (2.7) and (2.8) can be used to write (2.6) in the form

$$
K^{\rho, i}(\tilde{x})=\frac{K^{\rho, 0}(\tilde{x})}{\int_{0}^{d} v(y) d y} \int_{c}^{d} v(y)(y-\beta)^{(i)} d y, \quad 1 \leqq j<\sigma .
$$

An analogous argument establishes a dual relation between $K^{i, \sigma}$ and $K^{0, \sigma}, 1 \leqq i<\rho$.

The calculation of $K^{\rho, \sigma}$ is more complicated. If we apply Lemmas 1 and 2 to (2.5) and use the definition of $\psi$ we obtain

$$
\begin{aligned}
K^{\rho, \sigma}(\tilde{x}, \tilde{y})= & R \times S\left[(x-\tilde{x})^{(\rho-1)} \theta(\tilde{x}, x)(y-\tilde{y})^{(\sigma-1)} \theta(\tilde{y}, y)\right] \\
& -\theta(\tilde{y}, \beta) R\left[(x-\tilde{x})^{(\rho-1)} \theta(\tilde{x}, x)\right] \int_{c}^{d} v(y)(y-\tilde{y})^{(\sigma-1)} d y \\
& -\theta(\tilde{x}, \alpha) S\left[(y-\tilde{y})^{(\sigma-1)} \theta(\tilde{y}, y)\right] \int_{a}^{b} u(x)(x-\tilde{x})^{(p-1)} d x .
\end{aligned}
$$

We now simplify (2.9).

$$
\begin{aligned}
R \times S\left[(x-\tilde{x})^{(\rho-1)} \theta(\tilde{x}, x)(y-\tilde{y})^{(\sigma-1)} \theta(\tilde{y}, y)\right] \\
=\int_{a}^{z} u(x)(x-\tilde{x})^{(\rho-1)} d x \int_{c}^{\tilde{y}} v(y)(y-\tilde{y})^{(\sigma-1)} d y \\
\quad-\sum_{\mu=1}^{m} A_{\mu}\left(a_{\mu}-\tilde{x}\right)^{(\rho-1)} \theta\left(\tilde{x}, a_{\mu}\right) \sum_{\nu=1}^{n} B_{\nu}\left(b_{\nu}-\tilde{y}\right)^{(\sigma-1)} \theta\left(y, b_{\nu}\right) .
\end{aligned}
$$

From (2.8) and (2.1) we have

$$
\sum_{\mu=1}^{m} A_{\mu}\left(a_{\mu}-\tilde{x}\right)^{(\rho-1)} \theta\left(\tilde{x}, a_{\mu}\right)=\int_{a}^{\tilde{x}} u(x)(x-\tilde{x})^{(\rho-1)} d x+\frac{K^{\rho, 0}(\tilde{x})}{\int_{0}^{d} v(y) d y} .
$$

A similar argument shows

$$
\sum_{\nu=1}^{n} B_{\nu}\left(b_{\nu}-\tilde{y}\right)^{(\sigma-1)} \theta\left(\tilde{y}, b_{\nu}\right)=\int_{e}^{\tilde{y}} v(y)(y=\tilde{y})^{(\sigma-1)} d y+\frac{K^{0, \sigma}(\tilde{y})}{\int_{0}^{b} u(x) d x} .
$$


Using (2.11) and (2.12), we can write (2.10) in the form

$$
\begin{aligned}
R & \times S\left[(x-\tilde{x})^{(\rho-1)} \theta(\tilde{x}, x)(y-\tilde{y})^{(\sigma-1)} \theta(\tilde{y}, y)\right] \\
= & -\frac{K^{\rho, 0}(\tilde{x})}{\int_{c}^{d} v(y) d y} \int_{c}^{\tilde{y}} v(y)(y-\tilde{y})^{(\sigma-1)} d y-\frac{K^{0, \sigma}(\tilde{y})}{\int_{a}^{b} u(x) d x} \int_{a}^{\tilde{z}} u(x)(x-\tilde{x})^{(\rho-1)} d x \\
& -\frac{K^{\rho, 0}(\tilde{x}) K^{0, \sigma}(\tilde{y})}{\int_{a}^{b} u(x) d x \int_{c}^{d} v(y) d y} .
\end{aligned}
$$

Substitution of (2.13), (2.8) and the dual of (2.8) into (2.9) yields the form for $K^{\rho . \sigma}$ given in (2.5'). Q.E.D.

There is a natural relationship between the two basic Sard kernels $K^{p, 0}$ and $K^{0, \sigma}$ for $R \times S$ and the Peano kernels $K_{p-1,0}$ and $K_{0, \sigma-1}$ for $R$ and $S$. These properties are summarized in Corollary 1.

COROLLARY 1. If $r \in C^{\rho}[a, b]$, then

$$
R(r)=\int_{a}^{b} \frac{d^{p} r(x)}{d x^{\rho}} K_{p-1,0}(x) d x,
$$

where the Peano kernel $K_{p-1,0}$ for $R$ satisfies

$$
K_{p-1,0}(x)=K^{\rho, 0}(x) / \int_{c}^{d} v(y) d y .
$$

$A$ dual relationship exists between the Peano kernel $K_{0, \sigma-1}$ for $S$, and $K^{0, \sigma}$.

Proof. By Peano's Theorem [5, p. 70]

$$
R(r)=\int_{a}^{b} \frac{d^{p} r(x)}{d x^{\rho}} K_{p-1,0}(x) d x,
$$

where

$$
K_{p-1,0}(\tilde{x})=-R\left[(x-\tilde{x})^{(p-1)} \theta(\tilde{x}, x)\right] .
$$

The proof follows from the previous equation and the form given for $K^{\sigma, 0}$ in (2.5').

An analogous argument establishes a dual relationship between $K_{0, \sigma-1}$ and $K^{0 . \sigma}$. Q.E.D.

COROLLARY 2. Let (2.1) and (2.2) be Gaussian quadrature rules with nonnegative weight functions. Then $K^{2 m, 0}, K^{0,2 n}, F^{2 m, 0}$ and $G^{0,2 n}$ in Theorem 1 are nonnegative. If $\alpha=b$, then $K^{2 n, 2 n}$ is nonnegative.

Proof. By Corollary 1,

$$
K^{2 m, 0}(x)=K_{2 m-1,0}(x) \int_{0}^{d} v(y) d y .
$$

It is known [13, p. 61] that $K_{2 m-1,0}$ is nonnegative. Since $v$ is nonnegative, the previous equation implies $K^{2 m, 0}$ is nonnegative.

The definition of $F^{2 m, 0}$ implies

$$
\begin{aligned}
F^{2 m, 0}(\tilde{x}, \alpha) & =-\int_{a}^{\tilde{x}} u(x)(x-\tilde{x})^{(2 m-1)} d x, & & x<\alpha, \\
& =\int_{\tilde{z}}^{b} u(x)(x-\tilde{x})^{(2 m-1)} d x, & & x \geqq \alpha .
\end{aligned}
$$


$u(x) \geqq 0$ on $[a, b]$ implies $-u(x)(x-\tilde{x})^{(2 m-1)} \geqq 0$ on $[a, \tilde{x}]$ and $u(x)(x-\tilde{x})^{(2 m-1)} \geqq 0$ on $[x, b]$. Thus, $F^{2 m, 0}$ is nonnegative.

The same reasoning shows that $K^{0,2 n}$ and $G^{0,2 n}$ are nonnegative.

Let $\alpha=b$. By $\left(2.5^{\prime}\right)$ and Corollary 1 ,

$$
K^{2 m, 2 n}(x, y)=K_{0,2 n-1}(y)\left[F^{2 m, 0}(x, b)-K_{2 m-1,0}(x)\right]+K_{2 m-1,0}(x) G^{0,2 n}(y, \beta) .
$$

The definition of $F^{2 m, 0}$ implies

$$
F^{2 m, 0}(\tilde{x}, b)=-\int_{a}^{\tilde{x}} u(x)(x-\tilde{x})^{(2 m-1)} d x .
$$

Corollary 1 and $\left(2.5^{\prime}\right)$ give

$$
K_{2 m-1,0}(\tilde{x})=\sum_{\mu=1}^{m} A_{\mu}\left(a_{\mu}-\tilde{x}\right)^{(2 m-1)} \theta\left(\tilde{x}, a_{\mu}\right)-\int_{a}^{\tilde{x}} u(\tilde{x})(x-\tilde{x})^{(2 m-1)} d x .
$$

Thus,

$$
F^{2 m, 0}(x, b)-K_{2 m-1,0}(x)=-\sum_{\mu=1}^{m} A_{\mu}\left(a_{\mu}-x\right)^{(2 m-1)} \theta\left(x, a_{\mu}\right) .
$$

Since $A_{\mu}>0$ for $1 \leqq \mu \leqq m$ and $a<a_{1}<\cdots<a_{n}<b$, the previous equation implies that $F^{2 m, 0}-K_{2 m-1,0}$ is nonnegative. It follows from (2.14) that $K^{2 m, 2 n}$ is nonnegative. Q.E.D.

3. Error Bounds. The equality for $R \times S(f)$ given by $\left(2.4^{\prime}\right)$ can be the basis for infinitely many sharp bounds for the cross-product cubature error. One convenient bound [10, pp. 203-205], which uses the $L_{1}$ norm, is

$$
|R \times S(f)| \leqq M^{\rho, \sigma}(\alpha, \beta) N^{\rho, \sigma}(f ; a, \beta),
$$

where

$$
\begin{aligned}
M^{\rho, \sigma}(\alpha, \beta)= & \sum_{j<\sigma}\left\|K^{\rho, i}\right\|_{1}+\sum_{i<\rho}\left\|K^{i, \sigma}\right\|_{1}+\left\|K^{\rho, \sigma}\right\|_{1} \\
N^{\rho, \sigma}(f ; \alpha, \beta)=\max & \left\{\max _{a \leq x \leq b}\left|f_{\rho, j}(x, \beta)\right|, j<\sigma ;\right. \\
& \left.\max _{c \leq y \leq d}\left|f_{i, \sigma}(\alpha, y)\right|, i<\rho ; \max _{a \leq x \leq b ; e \leq y \leq d} \mid f_{\rho, \sigma}(x, y)\right\}_{\}} .
\end{aligned}
$$

By Theorem 1 and Corollary 1,

$$
\begin{aligned}
\left\|K^{p, i}\right\|_{1} & =\left|\int_{c}^{d} v(y)(y-\beta)^{(i)} d y\right|\left\|K_{\rho-1,0}\right\|_{1} \\
& =\left|\sum_{\nu=1}^{n} B_{\nu}\left(b_{\nu}-\beta\right)^{(i)}\right|\left\|K_{\rho-1,0}\right\|_{1}, \quad j<\sigma,
\end{aligned}
$$

and

$$
\begin{aligned}
\left\|K^{i, \sigma}\right\|_{1} & =\left|\int_{0}^{b} u(x)(x-\alpha)^{(i)} d x\right|\left\|K_{0, \sigma-1}\right\|_{1} \\
& =\left|\sum_{\mu-1}^{m} A_{\mu}\left(a_{\mu}-\alpha\right)^{(i)}\right|\left\|K_{0, \sigma-1}\right\|_{1}, \quad i<\rho .
\end{aligned}
$$


The Peano kernels $K_{\rho-1,0}$ and $K_{0, \sigma-1}$ do not depend on $\alpha$ and $\beta$. We can find the values of $\left\|K_{\rho-1,0}\right\|_{1}$ and $\left\|K_{0, \sigma-1}\right\|_{1}$ in [13] for most rules of interest. With these values, it is easy to calculate $\left\|K^{\rho, i}\right\|_{1}$ and $\left\|K^{i, \sigma}\right\|_{1}$ from (3.4) and (3.5).

It would be desirable to tabulate $\left\|K^{\rho, \sigma}\right\|_{1}$ for various cross-product rules to utilize (3.1) effectively. We can obtain a bound on $\left\|K^{\rho, \sigma}\right\|_{1}$ as follows: Theorem 1 and Corollary 1 imply

$$
\left\|K^{p, \sigma}\right\|_{1} \leqq\left\|K_{0, \sigma-1}\right\|_{1}\left[\left\|F^{p .0}\right\|_{1}+\left\|K_{p-1,0}\right\|_{1}\right]+\left\|K_{p-1,0}\right\|_{1}\left\|G^{0 . \sigma}\right\|_{1} .
$$

After interchanging the limits of integration, a direct calculation shows

$$
\left\|F^{\rho, 0}\right\|_{1} \leqq \int_{a}^{b}\left|u(x)(x-\alpha)^{(\rho)}\right| d x,
$$

and

$$
\left\|G^{0, \sigma}\right\|_{1} \leqq \int_{c}^{d}\left|v(y)(y-\beta)^{(\sigma)}\right| d y .
$$

Thus,

$$
\begin{aligned}
\left\|K^{\rho, \sigma}\right\|_{:} \leqq & \left\|K_{0, \sigma-1}\right\|_{1}\left[\int_{a}^{b}\left|u(x)(x-\alpha)^{(\rho)}\right| d x+\left\|K_{\rho-1,0}\right\|_{1}\right] \\
& +\left\|K_{\rho-1,0}\right\|_{1} \int_{c}^{d}\left|v(y)(y-\beta)^{(\sigma)}\right| d y .
\end{aligned}
$$

Relations (3.2), (3.4)-(3.6) imply

$$
\begin{aligned}
M^{\rho, \sigma}(\alpha, \beta) \leqq & \left\|K_{\rho-1,0}\right\|_{1}\left[\int_{c}^{d}\left|v(y)(y-\beta)^{(\sigma)}\right| d y+\sum_{i<\sigma}\left|\int_{c}^{d} v(y)(y-\beta)^{(i)} d y\right|\right] \\
& +\left\|K_{0, \sigma-1}\right\|_{1}\left[\int_{a}^{b}\left|u(x)(x-\alpha)^{(\rho)}\right| d x+\sum_{i<\rho}\left|\int_{a}^{b} u(x)(x-\alpha)^{(i)} d x\right|\right] \\
& +\left\|K_{\rho-1,0}\right\|_{1}\left\|K_{0, \sigma-1}\right\|_{1} .
\end{aligned}
$$

If the Peano kernels $K_{p-1,0}$ and $K_{0, \sigma-1}$ are nonnegative, then Corollary 1 implies

$$
\left\|K_{\rho-1,0}\right\|_{1}=R\left[x^{(\rho)}\right]
$$

and

$$
\left\|K_{0, \sigma-1}\right\|_{1}=S\left[y^{(\sigma)}\right] .
$$

If $K^{p, \sigma}$ is nonnegative, Theorem 1 gives

$$
\left\|K^{\rho, \sigma}\right\|_{1}=R \times S\left[(x-\alpha)^{(\rho)}(y-\beta)^{(\sigma)}\right] .
$$

Lemmas 1 and 2, and the previous equation imply

$$
\begin{aligned}
\left\|K^{\rho, \sigma}\right\|_{1} & =R\left[(x-\alpha)^{(\rho)}\right] \sum_{\nu=1}^{n} B_{\nu}\left(b_{\nu}-\beta\right)^{(\sigma)} \\
& =S\left[(y-\beta)^{(\sigma)}\right] \sum_{\mu=1}^{\infty} A_{\mu}\left(a_{\mu}-\alpha\right)^{(\rho)},
\end{aligned}
$$


provided $1 \leqq \rho<p+1,1 \leqq \sigma<q+1$. If $\rho=p+1$ and $\sigma=q+1$, then

$$
\begin{aligned}
\left\|K^{p, \sigma}\right\|_{1}= & \int_{0}^{b} u(x)(x-\alpha)^{(p+1)} d x \int_{c}^{d} v(y)(y-\beta)^{(\alpha+1)} d y \\
& -\sum_{\mu=1}^{m} A_{\mu}\left(a_{\mu}-\alpha\right)^{(p+1)} \sum_{\nu=1}^{n} B_{\nu}\left(b_{\nu}-\beta\right)^{(\alpha+1)} .
\end{aligned}
$$

If (2.1) and (2.2) are Gaussian rules with nonnegative weight functions, then relations (3.4), (3.5), (3.8)-(3.10) and Corollary 2 imply

$$
\begin{aligned}
M^{2 n, 2 m}(b, \beta)= & R\left[x^{(2 m)}\right] \sum_{i<2 n}\left|\int_{c}^{d} v(y)(y-\beta)^{(i)} d y\right| \\
& +S\left[y^{(2 n)}\right] \sum_{i<2 m}\left|\int_{a}^{b} u(x)(x-b)^{(i)} d x\right| \\
& +\int_{a}^{b} u(x)(x-b)^{(2 m)} d x \int_{c}^{d} v(y)(y-\beta)^{(2 n)} d y \\
& -\sum_{\mu=1}^{m} A_{\mu}\left(a_{\mu}-b\right)^{(2 m)} \sum_{\nu=1}^{n} B_{\nu}\left(b_{\nu}-\beta\right)^{(2 n)} .
\end{aligned}
$$

For certain purposes it may be convenient to replace some of the integrals in (3.7) and (3.11) by sums. Since (2.1) and (2.2) have precision $p$ and $q$, respectively,

$$
\int_{a}^{b} u(x)(x-\alpha)^{(i)} d x=\sum_{\mu=1}^{m} A_{\mu}\left(a_{\mu}-\alpha\right)^{(i)}, \quad i<\rho,
$$

and

$$
\int_{c}^{d} v(y)(y-\beta)^{(i)} d y=\sum_{\nu=1}^{n} B_{\nu}\left(b_{\nu}-\beta\right)^{(i)}, \quad j<\sigma .
$$

We can also write $R\left[x^{(2 m)}\right]=c_{m}$ and $S\left[y^{(2 n)}\right]=d_{n}$ in (3.11), where $c_{m}$ and $d_{n}$ are the constants in the classical error forms for (2.1) and (2.2):

$$
\begin{array}{ll}
R(r)=c_{m} \frac{d^{2 m} r(\xi)}{d x^{2 m}}, & \xi \in(a, b), \\
S(s)=d_{n} \frac{d^{2 n} s(\eta)}{d y^{2 n}}, & \eta \in(c, d) .
\end{array}
$$

4. Extension to Higher Dimensions. Theorem 1 can be extended to $n$ dimensions. Without giving all the details, we indicate the counterpart of relation $\left(2.4^{\prime}\right)$ in three dimensions.

The quadrature rules (2.1), (2.2) and

$$
\int_{0}^{f} w(z) t(z) d z=\sum_{\xi=1}^{0} C_{\xi} t\left(c_{\xi}\right)+T(t)
$$

generate a cross-product rule whose cubature error $R \times S \times T(f)$ is

$$
\begin{aligned}
R \times S \times T(f)= & \int_{0}^{f} \int_{0}^{d} \int_{a}^{b} u(x) v(y) w(z) f(x, y, z) d x d y d z \\
& -\sum_{\xi=1}^{0} \sum_{n=1}^{n} \sum_{\mu=1}^{\infty} A_{\mu} B_{v} C_{\xi} f\left(a_{\mu}, b_{\nu}, c_{\xi}\right) .
\end{aligned}
$$


Let $1 \leqq r+1$, where $r$ is the precision of (4.1), and let $\gamma \in[e, f]$. Sard's Kernel Theorem 74 [10, p. 290] gives the following counterpart of (2.4') for $R \times S \times T(f)$ :

$$
\begin{aligned}
R \times S \times T(f)= & \sum_{i<\sigma ; k<\tau} \int_{a}^{b} f_{\rho, i, k}(x, \beta, \gamma) K^{\rho, i, k}(x) d x \\
& +\sum_{i<\rho ; k<\tau} \int_{c}^{d} f_{i, \sigma, k}(\alpha, y, \gamma) K^{i, \sigma, k}(y) d y \\
& +\sum_{i<\rho ; j<\sigma} \int_{0}^{f} f_{i, i, \tau}(\alpha, \beta, z) K^{i, i, \tau}(z) d z \\
& +\sum_{k<\tau} \int_{c}^{d} \int_{a}^{b} f_{\rho, \sigma, k}(x, y, \gamma) K^{\rho, \sigma, k}(x, y) d x d y \\
& +\sum_{i<\rho} \int_{0}^{f} \int_{c}^{d} f_{i, \sigma, \tau}(\alpha, y, z) K^{i, \sigma, \tau}(y, z) d y d z \\
& +\sum_{i<\sigma} \int_{0}^{f} \int_{a}^{b} f_{\rho, i, \tau}(x, \beta, z) K^{\rho, i, \tau}(x, z) d x d z \\
& +\int_{0}^{f} \int_{0}^{d} \int_{a}^{b} f_{\rho, \sigma, \tau}(x, y, z) K^{\rho, \sigma, \tau}(x, y, z) d x d y d z .
\end{aligned}
$$

We find that all of the kernels in (4.2) are completely determined by the three basic kernels $K^{\rho, 0,0}, K^{0, \sigma, 0}$ and $K^{0,0, r}$. If $K_{\rho-1,0,0}, K_{0, \sigma-1,0}$ and $K_{0,0, \tau-1}$ denote the Peano kernels for the respective quadrature rules (2.1), (2.2) and (4.1), we find that

$$
\begin{aligned}
& K^{\rho, 0,0}(x)=K_{\rho-1,0,0}(x) \int_{c}^{d} v(y) d y \int_{c}^{f} w(z) d z, \\
& K^{0, \sigma, 0}(y)=K_{0, \sigma-1,0}(y) \int_{a}^{b} u(x) d x \int_{c}^{f} w(z) d z, \\
& K^{0,0, \tau}(z)=K_{0,0, \tau-1}(z) \int_{a}^{b} u(x) d x \int_{c}^{d} v(y) d y .
\end{aligned}
$$

Relations analogous to $\left(2.5^{\prime}\right)$ define the multivariable kernels in (4.2); explicit forms for the kernels in (4.2) can be found in [7].

5. Numerical Examples. To apply (3.1) we must calculate $M^{p, \sigma}(\alpha, \beta)$. It is interesting to compare the bound given by (3.7), with the actual value of $M^{p . \sigma}(\alpha, \beta)$.

Let (2.1) and (2.2) be the Gauss-Legendre one-point quadrature rule defined by $A_{1}=2, a_{1}=0, a=-1, b=1$. Since $p=q=1$, we may take $1 \leqq \rho, \sigma \leqq 2$. By direct calculation we find

$$
\begin{aligned}
& M^{1,1}(0,0)=5, \quad M^{2,1}(0,0)=3, \\
& M^{1,2}(0,0)=3 \text {, and } M^{2,2}(0,0)=13 / 9 \text {. }
\end{aligned}
$$

Relation (3.7) gives

$$
\begin{aligned}
M^{1,1}(0,0) \leqq 7, \quad M^{2,1}(0,0) \leqq 11 / 3 \\
M^{1,2}(0,0) \leqq 11 / 3, \quad \text { and } \quad M^{2,2}(0,0) \leqq 15 / 9 .
\end{aligned}
$$


By (3.11), $M^{2,2}(1,0)=22 / 9$. The bound obtained from $(3.7)$ is $M^{2,2}(1,0) \leqq 24 / 9$. The choice $(\alpha, \beta)=(0,0)$ forces some of the kernels in (3.2) to vanish. This observation explains why $M^{2,2}(0,0)<M^{2,2}(1,0)$.

Let (2.1) and (2.2) be the Gauss-Legendre two-point quadrature rule defined by $A_{1}=A_{2}=1, a_{1}=-\sqrt{ } 3 / 3, a_{2}=\sqrt{ } 3 / 3, a=-1, b=1$. If we approximate

$$
\int_{-1}^{1} \int_{-1}^{1} \ln (x+y+5)^{1 / 2} d x d y
$$

we find that the cross-product cubature error is 0.00017 . We obtain the following error bounds for (3.1) and (3.7):

$$
\begin{array}{ll}
\rho=\sigma=1 & 0.42 \\
\rho=\sigma=2 & 0.014 \\
\rho=\sigma=3 & 0.0079 .
\end{array}
$$

6. Acknowledgments. This work is a portion of the author's thesis for the Ph.D. degree in Mathematics at the University of Utah, written under the direction of Professor Robert E. Barnhill.

Department of Mathematics

The University of Utah

Salt Lake City, Utah 84112

1. A. C. AhLIN, "On error bounds for Gaussian cubature," SIAM Rev., v. 4, 1962, pp. 25-39. MR 27 \#2105.

2. R. E. BARNHILL, "An error analysis for numerical multiple integration. I," Math. Comp., v. 22, 1968, pp. 98-109. MR 37 \#2438.

3. M. M. CHAWLA, "On the estimation of errors of Gaussian cubature formulas," SIAM J. Numer. Anal., v. 5, 1968, pp. 172-181. MR 36 \#324.

4. P. J. DAvis, "Errors of numerical approximation for analytic functions," J. Rational Mech. Anal., v. 2, 1953, pp. 303-313. MR 14, 907. $28 \# 393$.

5. P. J. DAvis, Interpolation and Approximation, Blaisdell, Waltham, Mass., 1963. MR

6. I. A. EzRoH, "General forms of remainder terms of linear formulae in multi-dimensional approximate analysis. I, II," Mat. Sb., v. 38(80), 1956, pp. 389-416; v. 43(85), 1957, pp. 9-28. (Russian) MR 18, 32; MR 19, 1199.

7. F. G. LeTHER, Cross-Product Cubature Error Estimates, Ph.D. Thesis, The University of Utah, Salt Lake City, Utah, 1969.

8. J. N. LYNESS \& B. J. J. MCHUGH, "Integration over multidimensional hypercubes. I: A progressive procedure," Comput. J., v. 6, 1963, pp. 264-270.

9. S. M. NIKOL'sKIÍ, Quadrature Formulas, Fizmatgiz, Moscow, 1958. (Russian)

10. A. SARD, Linear Approximation, Math. Surveys, no. 9, Amer. Math. Soc., Providence, R.I., 1963.

11. D. D. Stancu, "The remainder of certain linear approximation formulas in two variables," J. Soc. Indust. Appl. Math. Ser. B Numer. Anal., v. 1, 1964, pp. 137-163. MR 31 \#1503.

12. F. STENGer, "Error bounds for the evaluation of integrals by repeated Gauss-type formulae," Numer. Math., v. 9, 1966, pp. 200-213, MR 34 \#5289.

13. A. H. STROUD \& D. SECREST, Gaussian Quadrature Formulas, Prentice-Hall, Englewood Cliffs, N. J., 1966. MR 34 \#2185. 